\title{
Neuroscience Engineering Education Using Self- learning by Mind-controlled LEGO Robots
}

\author{
Dean M. Aslam \\ Microsystems and Mind Lab, Electrical and Computer Engineering \\ Michigan State U., E. Lansing, MI 48824
}

I. Introduction: Unlike for physical health, individuals generally lack insight into their own cognitive condition. This blind spot, in addition to the stigma of seeking early help, results in many disorders related to neuroscience going untreated. Although many brain regions have been shown to play a role in cognitive processes, a comprehensive formalization of each region's contribution to cognition is still lacking. This paper, for the first time, envisions using a cognition-controlled programmable LEGO robot that will generate insights into the science of mental state of an individual through self-study and -learning approach as neuroscience and robotics are STEM fields of study. This self-study idea is unique to recent research in neuroscience and robotics for STEM education. The STEM areas involved are (a) computer, neuro, and data sciences, (b) fabrication, embedded system integration and sensor technologies, (c) electrical, mechanical, computer, and neural engineering, and (d), functions, density functions, and algorithms math. Algorithms based on EEG (Electroencephalogram) data coming from different parts of brain collected using fabric-embedded micro systems technology - such as in a cap or a shirt collar - help quantify neural activity for STEM education.

This paper focuses on neuroscience and robotics for STEM education for self-learning using programmable LEGO robots. Although, in the past, this research has developed creative STEM education modules as outreach part of NSF ERC for WIMS (Award Number EEC-9986866 during 2000 - 2010) [1][2][3], this is the first time that self-learning of neural problems for K-12 STEM education is investigated and described. The first ever scientific model of mind as an algorithm [4] developed by the author is the decision maker for STEM self-learning.

II. Cognition-controlled LEGO Robots for Neuroscience Engineering Self-Education: The author designed, built and tested LEGO robots controlled by measuring attentional focus using an algorithm that is based on alpha wave $(8-12 \mathrm{~Hz})$ and beta wave $(13-30 \mathrm{~Hz})$ fluctuations in neural activity. The mind-controlled LEGO robot moves if the user is focused on one thing and stops if the user thinks about many things [5]. Such a robot, shown in Fig. 1, uses mind's attention algorithm described in Fig. 1 (c). If $F(n)$ is FFT (Fast Fourier Transform) of EEG data with $\mathrm{N}$ points, the Power Spectral Density (PSD), P(n) = F(n) $\mathrm{F}^{*}(\mathrm{n}) / \mathrm{N}$, the attention level is defined by $\mathrm{R}$ $=E_{\alpha} / E_{\beta}[6]$ where $E_{\alpha}$ and $E_{\beta}$ are density functions defined in Fig. 1 (c). As shown in Fig. 1, cognition-controlled LEGO robot has two Microsystems: One in the cap as
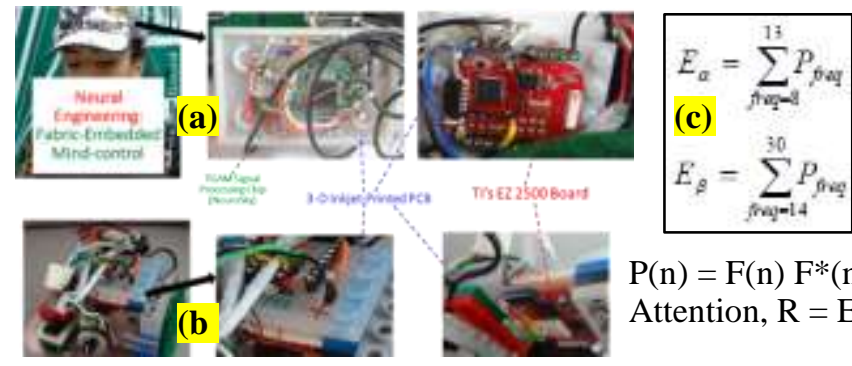

$\mathrm{P}(\mathrm{n})=\mathrm{F}(\mathrm{n}) \mathrm{F}^{*}(\mathrm{n}) / \mathrm{N}$; Attention, $\mathrm{R}=\mathrm{E}_{\alpha} / \mathrm{E}_{\beta}$

Fig. 1 Cap-mounted EEG sensor and signal processing Microsystem (a), a LEGO robot, with a second Microsystem, programmed to wirelessly respond to attention level of mind (b) and attention algorithm math (c). seen in Fig. 1(a) and the other in the robot as seen in Fig. 1(b). A $2.4 \mathrm{GHz}$ wireless connection is established between the two microsystems using TI's EZ2500 boards. NeuroSky's TGAM board, capable of providing 5 brainwaves, raw signal, attention level and meditation level, is used in the cap. The robot uses only attention level algorithm.

Unintentional mind wandering is linked to symptoms of depression, anxiety, and stress [7]. A combination of fMRI (functional Magnetic Resonance Imaging) and pupillometry study shows pupillary dilation mirroring brain patterns indicating repetitive thinking [8]. Such effects will lead to low attention and meditation levels. This can help define depression, anxiety, and stress (DAS) algorithms using the measured attention, R, and meditations, M, levels. One possible way to define DAS algorithm is to consider $\mathrm{Q}_{\mathrm{DAS}}=1 /(\mathrm{RxM})^{2}$ where $\mathrm{R}$ and $\mathrm{M}$ are measured using NeuroSky's single electrode EEG used for mind-controlled LEGO robot. However, a better set of experiments on self-study data collection can be achieved using BCI's EEG system shown in Fig. 2. In this case, EEG data from brain parts involved in DAS will be needed. 
A more accurate measure of anxiety will be the brainwaves detected from brain parts responsible for anxiety and other mental states shown in Fig 3. For example, the technology of mindcontrolled robot can be extended to develop empathy- and narcissism-controlled LEGO robots if the alpha and beta waves data coming from insula and Temporal Parietal Junction (TPJ) is measured and used to develop empathy algorithm. To accomplish this, the EEG data from TPJ and insula (Fig. 2) can be measured using BCI's 16 sensor system.

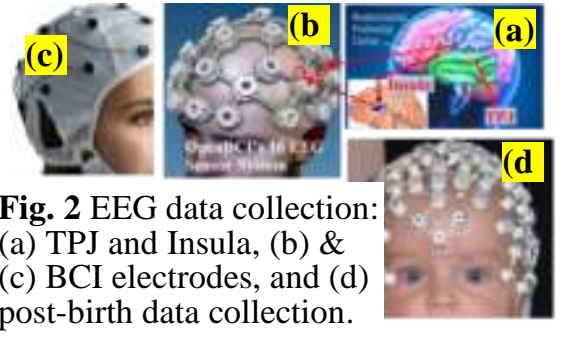

As a lack of empathy is associated with a variety of neural disorders, including narcissism, the goal of this research is to develop a robot able to detect the neural signatures of Empathy and other neural problems. To achieve this, it is important to build, program, and test LEGO robots controlled cognitive condition that will affect robot speed. The major challenge is to study neural activity from specific brain areas using EEG sensors and develop algorithms to analyze this activity and provide a read-out. Such studies can also be used to study post-birth neural problems using multi-electrode EEG on a baby's head as shown in Fig. 2. This approach is extendable to other mental states mentioned in Fig. 3, if the corresponding brain parts can

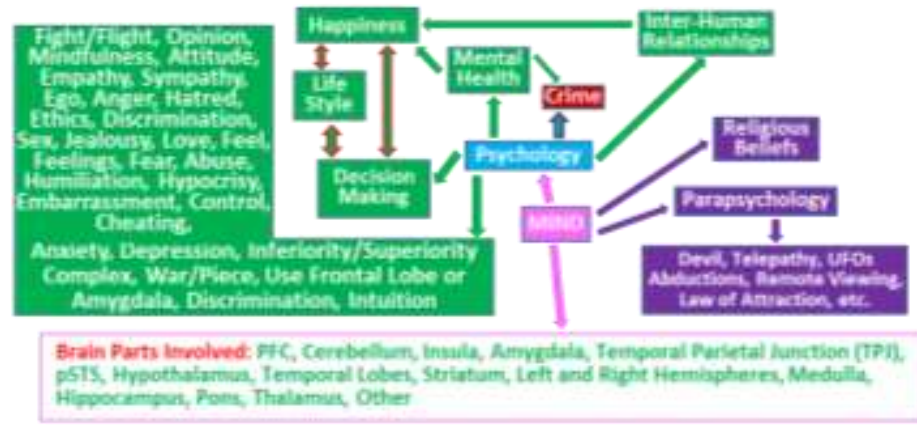

Fig. 3 Model of interrelated factors controlled by mind as MGBA-data-based algorithm [4]. be identified, to generate insights into cognitive processes using relatively inexpensive, highly adaptable commercial components such as OpenBCI's 16-sensor EEG. Commercial devices, similar to these concepts, are being used in mind-controlled games (Neurosky) [9][10][11]. This technology is applicable to in-home (a) brain/body health monitoring using smartphones and (b) self-study of psychological/neurological health problems. Algorithms for the prediction of depression [12] and other health problems [13] have also become the focus of recent research. However, the research in this paper extends this technology to some of the factors mentioned in Fig. 3.

\section{Fabric- and/or Tattoo-embedded Integrated Micro Systems (FTIMS):}

The self-study of psychological/neural problems focuses on (a) a holistic approach for mind/body data collection using FTIMS and smartphones (EEG signals along Microbiome-Gut-Brain-Axis (MGBA)), (b) thousands of years old meditation and herbal therapies and remedies that tend to cure the cause of health problems without the side effects [14], (c) the latest research on self-healing and (d) algorithm development using data from brain and/or MGBA. This research has tested FTIMS embedded in a cap, wig, jacket and shirt collar while scarf and tattoo embedded research is in progress. Some unique features of FTIMS, already tested, are (i) use of sowing machine to produce 50 micrometer $\mathrm{Cu}$ wire EEG electrodes as shown in Fig. 4 (f), (ii) 3D printed Microsystems as shown in Fig. 4 (g) \& (h) [15] and (iii) development of simple and preliminary algorithms for depression, anxiety, and stress (DAS)

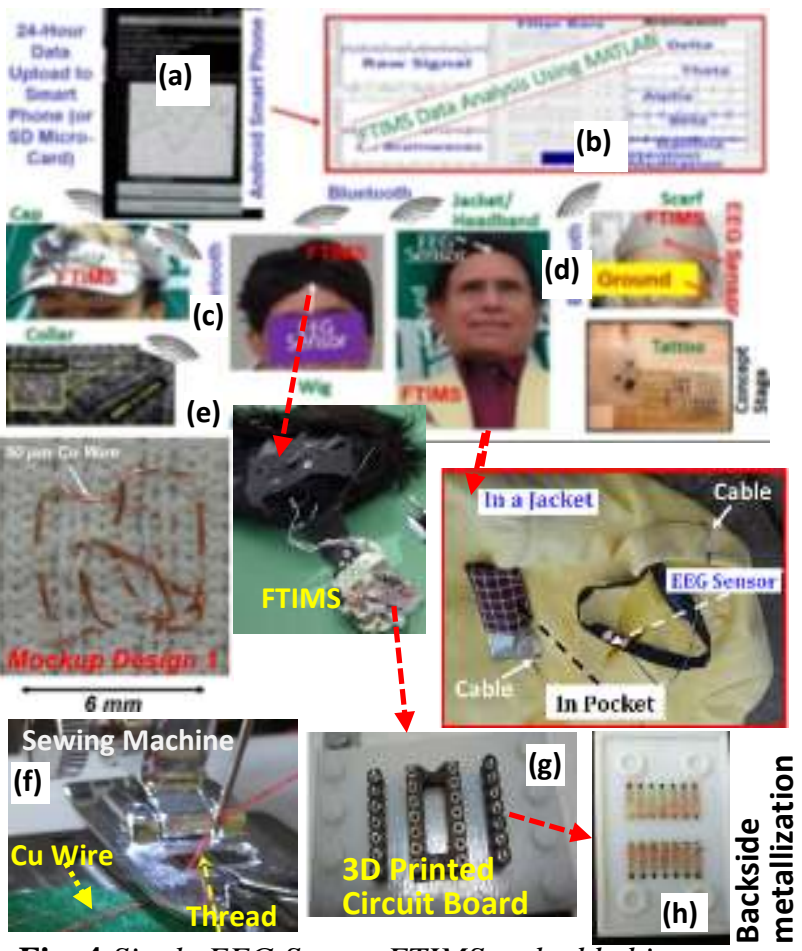

Fig. 4 Single EEG Sensor FTIMS embedded in a cap, wig, jacket, shirt collar, scarf or tattoo for data upload to smartphone $(a-d)$. Sewing machine used to get $\mathrm{Cu}$ wire electrodes (e-f). 3D printed circuit board (g). Backside metallization using a shadow mask (h). using attention and meditation algorithms as building blocks of preliminary models of DAS as mentioned in 
section II. The EEG signal, detected in the neck area (collar) was approx. $50 \%$ weaker than that on forehead.

The latest version FTIMS-based mind-controlled LEGO robot uses two EEG electrodes connected to Embedded Microsystem (EMS) as shown in Fig. 5: One activates the LEGO robot through a $2.4 \mathrm{GHz}$ wireless signal as shown in Fig. 5(a) \& (c) and the other shows attention and meditation level data on a smartphone connected through Bluetooth as shown in Fig. 5 (b \& d). The threshold attention level for robot to move can be adjusted by a potentiometer on a $0-100$ scale as shown in Fig. 5(c).

III. Amygdala Scrips: What happens to a human in the last 3-4 months in the womb, after birth and in the childhood has profound effect on personality, health and decision making. The childhood memories of or Amygdala Scripts (AS) [16][17], affecting profoundly the personality of humans, and prenatal environment [18][19], that also affects personality and health conditions, affect STEM and neural learning.

\section{Combination of fMRI and EEG Measurements}

Fig. 5 New Version of mind-controlled LEGO robot: Embedded MS (EMS) for robot control (a), EMS for brainwaves and attention/meditation displays (b), LEGO robot details (c), and brainwaves, attention \& meditation $(\mathrm{d})$

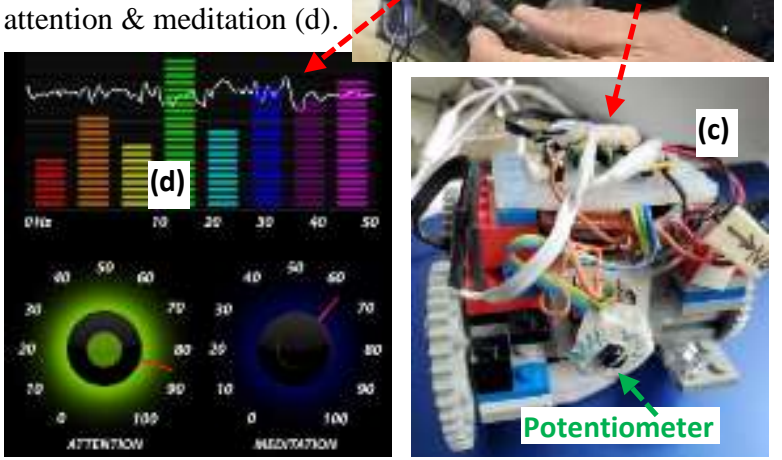

for EEG Data from Specific Brain Parts: An important aspect of this research, under investigation, is identifying the EEG signal coming from specific brain part/parts. For example, EEG signal coming from parts responsible for empathy is important for self-study of empathy related problems. Simultaneous EEG and fMRI measurement approach [20][21][22] can identify data from certain brain part or parts. Develop algorithm by finding FFT (Fast Fourier Transform) of relevant EEG data and test these algorithms under different conditions is very important.

Starting with empathy-controlled robot, other psychological/neural problems can be studied. The neuron firing in MGBA is also applicable to develop algorithms for factors mentioned in Fig. 3 that also shows parts of brain involved for development of algorithms for such factors. For example, empathy algorithm, involving insula and PFJ [23][24], should be possible using EEG data from PFJ and insula.

Starting with OpenBCI's 8- or 16-sensor EEG systems, as shown in Fig. 2, that is being studied, EEG signal will be acquired from PFJ and insula regions. Alpha and beta waves, from these regions, will be used to develop empathy algorithm using the FFT approach. Explore what regions of scull surface show higher EEG signal under anxiety, depression, and stress. Use simultaneous EEG and fMRI measurement approach [20][21][22] to identify data from a certain brain part or parts using systems to be acquired as shown in Fig. 6. fMRI can be used to identify parts of the brains involved in the factors mentioned in Fig. 3. The results will be reported in an upcoming publication.

V. Conclusions: This work reflects a creative and novel approach for recording and deciphering fundamental science of neural activity. Because one's mental state is not always accessible via introspection, this approach will enable a learner/observer/researcher to gain access to science of cognitive processes that may evade awareness. The LEGO robot will be able to provide a real-time read-out of neural activity accessible by EEG recording, making it possible to decipher the contributions of multiple brain areas across the cortex to mental states. Recent studies show that neuronal activity in Gut and communication of microbiomes with amygdala

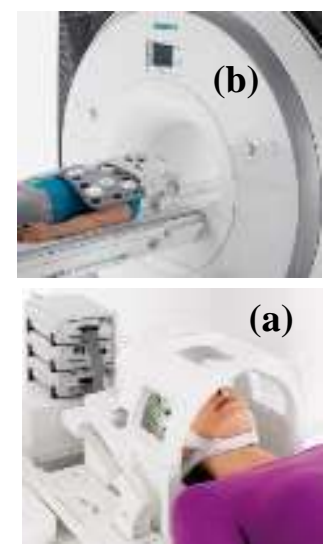

Fig. 6 EEG/fMRI systems; (a) Indiamart and (b) Siemens. is required for a complete cognitive study. Such an innovation, valuable for self-assessment of cognitive condition, is a valuable tool for researchers to study how brain areas act in concert to participate in a variety of cognitive processes including attention, learning, social cognition, and emotional states. Furthermore, use of fabric-embedded EEG sensors and microsystems will allow brain activity to be measured in naturalistic environments outside of a laboratory setting. Brain activity monitoring is also applicable to post-natal studies 
(Fig. 1). Simultaneous EEG/fMRI (functional Magnetic Resonance Imaging) can locate precise brain areas involved.

The STEM areas involved are (a) computer, neuro, and data sciences, (b) fabrication, embedded system integration and sensor technologies, (c) electrical, mechanical, computer, and neural engineering, and (d), functions, density function, and algorithms math. Mind-controlled LEGO robots will be used to explain neuroscience. The mind states of attention and meditation are neuroscience states. An overarching goal is to increase interest in (a) computer, neuro, and data sciences, (b) fabrication, embedded system integration and sensor technologies, (c) electrical, mechanical, computer, and neural engineering, and (d), functions, density function, and algorithms math.

\section{ACKNOWLEDGEMENTS}

The author is thankful to Mike Varney and Mustafa Alfatlawi for writing early versions of C-code for mind-controlled LEGO robot. The author is also thankful to Aseel Alinaizi, Ted Ransaw and Sara Steenrod for providing a useful feedback.

\section{REFERENCES}

[1] National Science Foundation center for Wireless Integrated Micro Systems (WIMS) was awarded to U of Michigan, Michigan State University and Michigan Technological University with Prof. Ken Wise as center Director, during 2000-2010.

[2] D.M. Aslam, "Creative Functionalized Bricks with Embedded Intelligence (FBEI) For Research-Oriented Provocative STEM and Workforce Learning", Int. J. Adv. Res. Comp. \& Comm. Eng., 9 (6), 2020;_DOI 10.17148/IJARCCE.2020.9605; https://ijarcce.com/wp-content/uploads/2020/06/IJARCCE.2020.9605.pdf

[3] D.M. Aslam, "Creative Functionalized Bricks with Embedded Intelligence (FBEI) For Research-Oriented Provocative STEM and Workforce Learning”, Keynote, 2021 IEEE Integrated STEM Education Conference, 13th March, 2021; https://ieeetv.ieee.org/channels/region-1/ieee-integrated-stem-conference-2021

[4] Dean M. Aslam, "Intriguing Aspects of New Scientific Mind Model as EEG Data Based Algorithm"; Int. J. Adv. Res. Comp. \& Comm. Eng., 8 (12), 2020; DOI 10.17148/IJARCCE.2019.81216; $\quad$ https://ijarcce.com/wpcontent/uploads/2020/01/IJARCCE.2019.81216.pdf

[5] Mind-controlled LEGO robot; http://www.egr.msu.edu/nsferc/40sec-Mindcontrol\%20Robot.mp4

[6] Ning-Han Liu, Cheng-Yu Chiang and Hsuan-Chin Chu, "Recognizing the Degree of Human Attention Using EEG Signals from Mobile Sensors" Sensors, 13, 10273-10286, 2013.

[7] P. Seli, R. E. Beaty, J. Marty-Dugas and D. Smilek, "Depression, Anxiety, and Stress and the Distinction Between Intentional and Unintentional Mind Wandering”, Psych. Consciousness: Theory, Research, and Practice, 6(2), 163-170, 2019: http://dx.doi.org/10.1037/cns0000182

[8] Makovac, Elena, Fagioli, Sabrina, Watson, David R et al. (4 more authors) "Response time as a proxy of ongoing mental state : A combined fMRI and pupillometry study in Generalized Anxiety Disorder", Neuroimage, 380-391, 2019: https://doi.org/10.1016/i.neuroimage.2019.02.038

[9] J. Esquicha-Tejada, S. Pari-Larico, B. Llerena-Urday, Á. Fernández del Carpio, K. Rosas-Paredes, "Interactive Toy to Strengthen the Memory, Attention and Logic of Primary Education Students Using Sphero, Arduino and Neurosky Mindwave EEG"; http://ceur-ws.org/Vol-2747/paper23.pdf

[10] A. Lewis Brooks, S. Brahman, B. Kapralos, A. Nakajima, J. Tyerman and L.C. Jain "Recent Advances in Technologies for Inclusive Well-Being Virtual Patients, Gamification and Simulation", 2021; https://link.springer.com/book/10.1007\%2F978-3030-59608-8\#editorsandaffiliations

[11] G. A. Mendes, V. Icon \& L. Cunha de Miranda, Icon, "Brain-Computer Interface Games Based on Consumer-Grade EEG Devices: A Systematic Literature Review”, International J. Human-Computer Interfaces, 36 (2), 105-142, 2020.

[12] J. Wang, J. Sareen, Scott Patten, J. Bolton, N. Schmitz, A. Birney, "A prediction algorithm for first onset of major depression in the general population: development and validation", J Epidemiol Community Health, 1-7, 2014.

[13] H-J Hwang, S. Kim, S. Choi and C-H. Im, "EEG-Based Brain-Computer Interfaces: A Thorough Literature Survey", International J. Human-Computer Interfaces, 9(12), 814-826, 2013; https://doi.org/10.1080/10447318.2013.780869

[14] D.M. Aslam, "Self-Study and -Care of Human Health Problems Guided by New Scientific Mind Model", Int. J. Adv. Research Computer \& Comm. Engin, (IJARCCE), 10 (5), 2021; DOI 10.17148/IJARCCE.2021.10502

[15] A. Alforidi, D.M. Aslam, D. Li and X. Liang, "3D - Printed Multilayer Processes for Lightweight Wearable and Flexible Microsystems", IJAR, vol. 7, 742-745, 2019, http://dx.doi.org/10.21474/IJAR01/10203

[16] Stokes, Tim, "What Freud Did Not Know', Rutgers University Press, 2013 (Amygdala Scripts mentioned for the first time in this book); https://www.psychologytoday.com/us/blog/what-freud-didnt-know/201002/taming-your-personal-rogues

[17] Dante Cicchetti, "An Odyssey of Discovery: Lessons Learned through Three Decades of Research on Child Maltreatment", American Psychologist, 59(8), 731-741353, 2004.

[18] J. A. Mennella, C. P. Jagnow, and G. K. Beauchamp "Prenatal and Postnatal Flavor Learning by Human Infants", Pediatrics, 107(6): E88., 2001. doi: 10.1542/peds.107.6.e88; https://www.ncbi.nlm.nih.gov/pmc/articles/PMC1351272/pdf/nihms5608.pdf 


\section{International Journal of Advanced Research in Computer and Communication Engineering}

Vol. 10, Issue 7, July 2021

DOI 10.17148/IJARCCE.2021.10701

[19] T.L. Nicole et al., "Exposure to prenatal phthalate mixtures and neurodevelopment in the Conditions Affecting Neurocognitive Development and Learning in Early childhood (CANDLE) study", https://doi.org/10.1016/j.envint.2021.106409

[20] S.D. Jackob N. Keynan, Yehudit Meir-Hasson, Gadi Gilam, Avihay Cohen, Gilan Jackont, Sivan Kinreich, Limor Ikar, Ayelet Or-Borichev, Amit Etkin, Anett Gyurak, Ilana Klovatch, Nathan Intrator, and Talma Hendlernm, "Limbic Activity Modulation Guided by Functional Magnetic Resonance Imaging-Inspired Electroencephalography Improves Implicit Emotion Regulation", Biological Psychiatry, 2016; 80:490-496 www.sobp.org/journal

[21] M.S. George, "Is Functional Magnetic Resonance Imaging-Inspired Electroencephalogram Feedback the Next New Treatment in Psychiatry?", Biological Psychiatry, 2016; 80:422-423 www.sobp.org/journal

[22] V. Zotev, R. Phillips, H. Yuan, M. Misaki, J. Bodurka, "Self-regulation of human brain activity using simultaneous real-time fMRI and EEG neurofeedback", NeuroImage, 85, 985-995, 2014.

[23] Tania Singer, Hugo D. Critchley and Kerstin Preuschoff, "A common role of insula in feelings, empathy and uncertainty", Trends in Cognitive Sciences, 13 (8), 2009.

[24] J. Zaki and K. Ochsner "The neuroscience of empathy: progress, pitfalls and promise", Nature Neuroscience, 15 (5), 2012. 